\title{
Dietary patterns among a national random sample of British adults
}

\author{
J A Pryer, R Nichols, P Elliott, B Thakrar, E Brunner, M Marmot
}

\begin{abstract}
Department of Primary Care and Population Sciences, Royal Free and University College Medical School, University College London, Royal Free Campus, Rowland Hill Street, London NW3 2PF, UK

J A Pryer
\end{abstract}

Department of Epidemiology and Public Health, Imperial College School of Medicine, London

R Nichols

P Elliott

Department of Epidemiology and Population Health, London School of Hygiene and Tropical Medicine

B Thakrar

Department of Epidemiology and Public Health, Royal Free and University College Medical School, University College London

E Brunner

M Marmot

Correspondence to: Dr Pryer (j.pryer@ucl.ac.uk)

Accepted for publication 17 July 2000

\begin{abstract}
Study objectives-To identify groups within the UK male and female population who report similar patterns of diet. Design-National representative dietary survey, using seven day weighed dietary records, of men and women aged 16-64 years living in private households in Great Britain in 1986-7. Cluster analysis was used to aggregate participants into diet groups.

Setting-Great Britain.

Participants-1087 men and 1110 women. Results-93\% of men and $86 \%$ of women fell into one of four distinct diet groups. Among men the most prevalent diet group was "beer and convenience food" (34\% of the male population); second was "traditional British diet" (18\%); third was "healthier but sweet diet" (17.5\%) and fourth was "healthier diet " (17\%). Among women, the most prevalent diet group was " traditional British diet" (32\%); second, was "healthy cosmopolitan diet" (25\%); third was a "convenience food diet" (21\%); and fourth was "healthier but sweet diet" (15\%). There were important differences in nutrient profile, sociodemographic and behavioural characteristics between diet groups.

Conclusions-Cluster analysis identified four diet groups among men and four among women, which differed not only in terms of reported dietary intakes, but also with respect to nutrient, social and behavioural profiles. The groups identified could provide a useful basis for development, monitoring and targeting of public health nutrition policy in the UK.

(F Epidemiol Community Health 2001;55:29-37)
\end{abstract}

Empirical dietary surveys frequently examine the food intake of a population in terms of nutrient intake. This approach provides valuable information on nutrient adequacy and excess and allows testing of a priori hypotheses on the association between nutrients and morbidity. However, the approach does not allow the complexities of dietary intake of individuals, or groups of individuals, to be considered in terms of their overall dietary pattern.

In recent years there has been increasing interest in the identification of dietary patterns as consumed by populations. It has been suggested that such analyses could shed light on the complex relation between diet and chronic disease. ${ }^{1-3}$ From a public health perspective, identification of groups within a population that consume similar patterns of diet would be of value to policy makers for translating national dietary goals into practical dietary recommendations for the public, for monitoring population trends towards nutritionally "healthier" diets, for identification and surveillance of those at nutritional risk, and for tailoring and targeting public health nutritional interventions.

Previous approaches to an analysis of dietary patterns have used frequency of food use to develop food variety scores or a qualitative food use profile of a population. ${ }^{245}$ More recently, multivariate statistical techniques have been used to examine the combination of foods consumed by populations, relating these either to population characteristics, or morbidity. Several studies have used factor analysis to classify dietary patterns according to the frequency of reported food consumption ${ }^{2}{ }^{6}$ or reported food intake. ${ }^{7-9}$ However, the factors identified by this technique do not refer to identifiable groups of individuals within a population, and hence do not give an indication of the prevalence of a particular type of diet. Cluster analysis on the other hand, aims to identify relatively homogeneous groups within the population based upon selected attributes (dietary variables). The technique has not been widely used to analyse dietary data.

This study was undertaken to explore whether relatively homogeneous groups reporting similar patterns of diet could be identified in a representative sample of British men and women. A further aim was to examine the nutrient profile, and sociodemographic characteristics associated with the groups.

\section{Methods}

The database used was the Dietary and Nutritional Survey of British Adults (DNSBA). The conduct and results of the survey have been described in detail elsewhere. ${ }^{10}$ Briefly fieldwork was carried out between October 1986 and August 1987. The sample was recruited using a multi-stage random probability design. The electoral roll was used as the sampling frame. The frame was stratified by region and within each major stratum, electoral wards were ranked by the proportion of heads of households in different socioeconomic groups using census data. A total of 120 wards were selected as first stage units, with probability proportional to the total electorate in each ward. In each ward, 33 addresses were selected and one person from each household was selected using the technique developed by Kish. ${ }^{11}$ In total, 1087 men and 1110 women aged 16-64 years completed the full dietary survey, a response rate of 70 per cent. ${ }^{10}$ Data 
Table 1 Median dietary intakes ( $\mathrm{g} /$ week or $\mathrm{ml} /$ week) for four large clusters (and the relative level ${ }^{\star}$ for all men combined): men

\begin{tabular}{|c|c|c|c|c|c|c|c|c|c|}
\hline \multirow{3}{*}{$\begin{array}{l}\text { Food group } \\
\text { Pasta/rice }\end{array}$} & \multirow{3}{*}{$\begin{array}{l}\begin{array}{l}\text { Male } \\
\text { population } \\
\text { median }\end{array} \\
163\end{array}$} & \multicolumn{8}{|c|}{ Large clusters } \\
\hline & & \multicolumn{2}{|c|}{$\begin{array}{l}\text { MC1 } \\
\text { Convenience/diet } \\
(n=366) \text { median }\end{array}$} & \multicolumn{2}{|c|}{$\begin{array}{l}\text { MC2 } \\
\text { Traditional } \\
(n=192) \text { median }\end{array}$} & \multicolumn{2}{|c|}{$\begin{array}{l}\text { MC3 } \\
\text { Mixed/sweet } \\
(n=190) \text { median }\end{array}$} & \multicolumn{2}{|c|}{$\begin{array}{l}\text { MC4 } \\
\text { Healthier } \\
(n=185) \text { median }\end{array}$} \\
\hline & & 181 & MH & 66 & $\mathrm{~L}$ & 163 & MH & 200 & MH \\
\hline White bread/refined cereals & 538 & 536 & MH & 1066 & $\mathrm{H}$ & 472 & ML & 370 & ML \\
\hline Wholegrain bread/cereals & 258 & 74 & $\mathrm{~L}$ & 100 & $\mathrm{~L}$ & 479 & $\mathrm{H}$ & 562 & $\mathrm{H}$ \\
\hline Cakes/pastries/puddings/biscuits & 482 & 278 & ML & 515 & $\mathrm{MH}$ & 1065 & $\mathrm{H}$ & 441 & ML \\
\hline High fat dairy & 1222 & 1106 & ML & 1676 & MH & 1695 & MH & 718 & ML \\
\hline Low fat dairy & 0 & 0 & $\mathrm{~L}$ & 0 & $\mathrm{~L}$ & 64 & $\mathrm{~L}$ & 921 & $\mathrm{~L}$ \\
\hline Eggs & 152 & 135 & ML & 165 & ML & 178 & MH & 162 & MH \\
\hline Butter & 15 & 10 & ML & 40 & $\mathrm{H}$ & 10 & ML & 10 & ML \\
\hline Poly/low fat spreads & 0 & 0 & $\mathrm{~L}$ & 0 & $\mathrm{~L}$ & 43 & $\mathrm{~L}$ & 39 & $\mathrm{~L}$ \\
\hline Other margarines & 10 & 20 & $\mathrm{H}$ & 24 & $\mathrm{H}$ & 0 & $\mathrm{~L}$ & 0 & $\mathrm{~L}$ \\
\hline Bacon $/ \mathrm{ham}$ & 104 & 81 & ML & 136 & $\mathrm{MH}$ & 92 & $\mathrm{ML}$ & 117 & $\mathrm{MH}$ \\
\hline Beef/veal/lamb/pork & 379 & 402 & MH & 434 & $\mathrm{MH}$ & 374 & ML & 340 & ML \\
\hline $\begin{array}{l}\text { Poultry } \\
\text { Pons }\end{array}$ & 145 & 159 & $\mathrm{MH}$ & 106 & ML & 145 & ML & 170 & MH \\
\hline Prepared meat products & 368 & 476 & MH & 417 & MH & 295 & ML & 240 & ML \\
\hline Fish/shellfish & 15 & 0 & 0 & 0 & 0 & 66 & $\mathrm{H}$ & 137 & $\mathrm{H}$ \\
\hline Vegetables/salad & 1523 & 1298 & ML & 1755 & $\mathrm{MH}$ & 1602 & $\mathrm{MH}$ & 1600 & $\mathrm{MH}$ \\
\hline Chips/roast potatoes & 428 & 504 & $\mathrm{MH}$ & 489 & $\mathrm{MH}$ & 370 & ML & 296 & ML \\
\hline Fruit/nuts & 291 & 96 & $\mathrm{~L}$ & 305 & MH & 533 & $\mathrm{H}$ & 487 & $\mathrm{H}$ \\
\hline Sugar/confectionery & 222 & 182 & ML & 357 & $\mathrm{H}$ & 256 & $\mathrm{MH}$ & 156 & ML \\
\hline Fruit juices & 0 & 0 & $\mathrm{~L}$ & 0 & $\mathrm{~L}$ & 0 & $\mathrm{~L}$ & 263 & $\mathrm{~L}$ \\
\hline Soft drinks & 311 & 275 & ML & 235 & ML & 344 & $\mathrm{MH}$ & 330 & $\mathrm{MH}$ \\
\hline Liquers/spirits/wines & 0 & 0 & $\mathrm{~L}$ & 0 & $\mathrm{~L}$ & 38 & $\mathrm{~L}$ & & $\mathrm{~L}$ \\
\hline Beer/cider & 1311 & 2023 & $\mathrm{H}$ & 1058 & ML & 718 & $\mathrm{~L}$ & 1522 & $\mathrm{MH}$ \\
\hline Coffee tea & 1568 & 1001 & ML & 1471 & ML & 2021 & $\mathrm{MH}$ & 1919 & $\mathrm{MH}$ \\
\hline Tea & 3297 & 2685 & ML & 5653 & $\mathrm{H}$ & 3301 & MH & 3288 & $\mathrm{MH}$ \\
\hline
\end{tabular}

${ }^{\star} \mathrm{L}=\mathrm{Low}<50 \%$ median; $\mathrm{ML}=$ Moderate low $=50-99 \% ; \mathrm{MH}=$ moderate high $=100-149 \% ; \mathrm{H}=$ high $>150 \%$.

were collected in four rounds (autumn, winter, spring, summer) to ensure that seasonality effects do not bias estimates from the aggregated data. ${ }^{10}$

Study participants were issued with a calibrated dietary weighing scales and asked to keep a weighed record of all foods consumed both in and out of the home over a seven day period.

Body weight and height were measured in standard fashion. Information on age, social class, ethnic origin, geographical area of residence, mineral and vitamin supplement use, cigarette smoking, and whether the food diary was affected by the participant being on a slimming diet, or due to illness during the week of the study, were also collected using an interviewer administered questionnaire. A copy of the data was obtained from The Office of Population, Census, and Surveys, and checked by replicating selected analyses and by undertaking range checks. ${ }^{10}$

The clustering technique used was a hierarchical agglomerative (or stepwise) technique available on SPSSx. The aim of the hierarchical agglomerative clustering algorithms is to find the most efficient step at each stage in a progressive synthesis of a population from " $n$ " cases to "N" groups according to predefined criteria, based upon a measure of distance or similarity. One source of variation in the hierarchical clustering techniques is in choice of distance or similarity criteria used. Ward's method was used as suggested by Everitt. ${ }^{12}$ Ward's method uses the squared Euclidean distance and is based upon the proposition that at any stage in the analysis the loss of information resulting from the grouping of entities into clusters can be measured by the total sum of squared deviations of each point from the mean of the cluster to which they belongs. At each step of the analysis the union of every possible pair of clusters is considered and the two clusters whose fusion results in the minimum increase in the error sum of squares are combined. ${ }^{12}$ In Monte Carlo studies, Ward's method has been found to be the most robust clustering method using a similarity matrix based upon squared Euclidean distances. ${ }^{13}{ }^{14}$ One major advantage of hierarchical clustering algorithms is that results are presented in the form of a dendogram. This aids the investigator in exercising judgements on the number of clusters that exist or are useful for the purpose at hand.

Analyses were conducted for men and women separately because we expected that women and men had different eating habits. Fifty one food/beverage groups were used in the analysis. We choose food variables because we wanted to identify food pattern clusters. Continuous food and beverage group values (g/week or ml/week) were standardised by converting to the standard normal deviate. This is to ensure that clusters are not influenced by food categories with high specific gravity (for example, beverages). A matrix of distance coefficients based upon the squared Euclidean distance was computed. A stepwise fusion of cases based upon the distance coefficient matrix was then produced. The clustering coefficient was used to indicate the stage on the agglomeration schedule where large changes between fusions were evident as compared with immediately preceding stages. ${ }^{12}$ In tabular presentation in this paper, the 51 food/drink groups defined by $\mathrm{MAFF}^{10}$ have been aggregated into 25 food/drink groups for presentation. To test the stability of the solution obtained, two split samples were used and eight large clusters and four small clusters were identified in each. ${ }^{12}$

The survey chose to use the seven day weighed intake method because of the detail 
Table 2 Median dietary intakes (g/week or $\mathrm{ml} /$ week) for four large clusters (and the relative level ${ }^{\star}$ for all women combined): women

\begin{tabular}{|c|c|c|c|c|c|c|c|c|c|}
\hline \multirow{3}{*}{$\begin{array}{l}\text { Food group } \\
\text { Pasta/rice }\end{array}$} & \multirow{3}{*}{$\begin{array}{l}\begin{array}{l}\text { Female } \\
\text { population } \\
\text { median }\end{array} \\
112\end{array}$} & \multicolumn{8}{|c|}{ Large clusters } \\
\hline & & \multicolumn{2}{|c|}{$\begin{array}{l}\text { FC1 } \\
\text { Traditional } \\
(n=355) \text { median }\end{array}$} & \multicolumn{2}{|c|}{$\begin{array}{l}F C 2 \\
\text { Healthier/ } \\
\text { cosmopolitan } \\
(n=275) \text { median }\end{array}$} & \multicolumn{2}{|c|}{$\begin{array}{l}\text { FC3 } \\
\text { Convenience } \\
(n=229) \text { median }\end{array}$} & \multicolumn{2}{|c|}{$\begin{array}{l}\text { FC4 } \\
\text { Healthier/sweet } \\
(n=168) \text { median }\end{array}$} \\
\hline & & 82 & ML & 164 & MH & 132 & MH & 85 & ML \\
\hline White bread/refined cereals & 305 & 489 & $\mathrm{H}$ & 134 & $\mathrm{~L}$ & 353 & MH & 174 & ML \\
\hline Wholegrain bread/cereals & 266 & 157 & ML & 480 & $\mathrm{H}$ & 113 & $\mathrm{~L}$ & 529 & $\mathrm{MH}$ \\
\hline Cakes/pastries/puddings/biscuits & 423 & 445 & $\mathrm{MH}$ & 390 & ML & 320 & ML & 602 & $\mathrm{MH}$ \\
\hline High fat dairy & 913 & 1372 & $\mathrm{H}$ & 658 & ML & 704 & ML & 615 & ML \\
\hline Low fat dairy & 143 & 0 & $\mathrm{~L}$ & 390 & $\mathrm{H}$ & 16 & $\mathrm{~L}$ & 1036 & $\mathrm{H}$ \\
\hline Eggs & 105 & 108 & $\mathrm{MH}$ & 114 & $\mathrm{MH}$ & 82 & ML & 106 & $\mathrm{MH}$ \\
\hline Butter & 15 & 28 & $\mathrm{H}$ & 10 & ML & 10 & ML & 10 & ML \\
\hline Poly/low fat spreads & 0 & 0 & $\mathrm{~L}$ & 17 & $\mathrm{~L}$ & 0 & $\mathrm{~L}$ & 88 & $\mathrm{~L}$ \\
\hline Other margarines & 0 & 14 & $\mathrm{~L}$ & 0 & $\mathrm{~L}$ & 10 & $\mathrm{~L}$ & 0 & $\mathrm{~L}$ \\
\hline Bacon/ham & 62 & 74 & $\mathrm{MH}$ & 46 & ML & 45 & ML & 75 & $\mathrm{MH}$ \\
\hline Beef/veal/lamb/pork & 260 & 257 & ML & 326 & $\mathrm{MH}$ & 198 & ML & 254 & ML \\
\hline Poultry & 106 & 78 & ML & 146 & $\mathrm{MH}$ & 100 & ML & 98 & ML \\
\hline Prepared meat products & 202 & 247 & MH & 138 & ML & 220 & $\mathrm{MH}$ & 163 & ML \\
\hline Fish/shellfish & 41 & 0 & $\mathrm{~L}$ & 104 & $\mathrm{H}$ & 0 & $\mathrm{~L}$ & 85 & $\mathrm{H}$ \\
\hline Vegetables/salad & 1179 & 1168 & ML & 1335 & MH & 845 & ML & 1409 & $\mathrm{MH}$ \\
\hline Chips/roast potatoes & 230 & 331 & $\mathrm{MH}$ & 100 & $\mathrm{~L}$ & 292 & $\mathrm{MH}$ & 176 & ML \\
\hline Fruit/nuts & 389 & 250 & ML & 686 & $\mathrm{H}$ & 187 & $\mathrm{~L}$ & 716 & $\mathrm{H}$ \\
\hline Sugar/confectionery & 143 & 206 & $\mathrm{MH}$ & 95 & ML & 164 & $\mathrm{MH}$ & 126 & ML \\
\hline Fruit juices & 0 & 0 & $\mathrm{~L}$ & 296 & $\mathrm{~L}$ & 0 & $\mathrm{~L}$ & 36 & $\mathrm{~L}$ \\
\hline Soft drinks & 336 & 298 & ML & 302 & ML & 788 & $\mathrm{H}$ & 176 & ML \\
\hline Liquers/spirits/wines & 34 & 0 & $\mathrm{~L}$ & 20 & $\mathrm{H}$ & 0 & $\mathrm{~L}$ & 30 & ML \\
\hline Beer/cider & 0 & 0 & $\mathrm{~L}$ & 0 & $\mathrm{~L}$ & 0 & $\mathrm{~L}$ & 0 & $\mathrm{~L}$ \\
\hline Coffee & 1717 & 1212 & ML & 2352 & MH & 2002 & $\mathrm{MH}$ & 2002 & $\mathrm{MH}$ \\
\hline Tea & 3299 & 4130 & $\mathrm{MH}$ & 2796 & ML & 4552 & $\mathrm{MH}$ & 4552 & MH \\
\hline
\end{tabular}

${ }^{\star} \mathrm{L}=$ Low <50\% median; $\mathrm{ML}=$ Moderate low $=50-99 \% ; \mathrm{MH}=$ moderate high $=100-149 \% ; \mathrm{H}=$ high $>150 \%$.

and precision needed. This method has the advantage of being the most precise method available for accurately recording the amounts of food consumed. The method does not have the disadvantage of inaccuracies attributable to errors in recall and errors involved in estimating portion size are also minimised. However, validation studies of the weighed intake that have used either doubly labelled water to assess energy expenditure, or urinary nitrogen as a marker for dietary nitrogen intake, indicate that underreporting bias is evident especially at the lower end of the reported intake. ${ }^{15}$ Also, in population-based surveys, there is evidence of underreporting in obese people and among women to a greater degree than among men. ${ }^{15}$

The proportion of "low energy reporters" by dietary cluster is presented for information. For the purposes of this study, a reported dietary energy intake of less than 1.2 estimated basal metabolic rate (BMR) was taken as indicative of "low energy reporting" (LER). ${ }^{15}$ $\mathrm{BMR}$ was estimated using predictive equations that use age, sex and body weight. ${ }^{16}$

Statistical comparisons were made across the clusters in terms of reported food group consumption, macro-nutrient and micronutrient intakes and selected socioeconomic, demographic and behavioural variables. Nonparametric Kruskal-Wallis one way analysis of variance was used to test for differences between clusters in food consumption and alcohol. Parametric one way analysis of variance was used to test for between group differences in reported nutrient intake. Some variables were transformed using natural logarithms before statistical analysis. The $\chi^{2}$ test was used to test for between group differences in the frequency distribution of categorical variables; where the expected cell size was under five, Fisher's exact test was used. Statistical analyses were performed using the SPSSx and SAS statistical packages. Tables present for men and women respectively median food/ beverage intakes because men and women's intakes were different. Cluster medians food/ beverage intakes below $50 \%$ of the male and female median intake were considered "low". Cluster median intakes between $50-99 \%$ and $100-149 \%$ were considered respectively "moderate-low" and "moderate-high", and intakes of $150 \%$ or more of the male and female median intake were considered "high". A list of the 51 food/beverage groups is shown in the appendix..

\section{Results}

IDENTIFICATION OF DIET CLUSTERS

Among both men and women, four large clusters were identified comprising 93 per cent and 86 per cent of the sample respectively, and there were four small clusters. Results will be presented here for the four large clusters only for men and women. The level of agreement between split samples was the same. In conclusion the clusters were robust.

MEN

The most prevalent male dietary group in men was cluster 1 (MC1) ( $n=366 ; 34 \%$ of men). This had reported median food/drink groups in the "high" for: beer/cider, and other margarines, and moderately high for: prepared meat, meat products, chips and white bread. Food groups in the "low" category included: wholegrain cereals, fruits and nuts. Cluster median intakes were zero for polyunsaturated fats, low fat dairy products, fish/shellfish, fruit juices, liqueurs/spirits/wines (table 1). This dietary pattern could be considered a "convenience food/beer" diet high in meat products, chips, and beer .

Male cluster 2 (MC2) ( $n=192 ; 18 \%$ of men) was the second most prevalent group with 
Table 3 Energy intake, body mass index, "low energy reporters" self reported slimming: male and female populations and four large male and female dietary clusters

\begin{tabular}{|c|c|c|c|c|c|c|c|c|c|c|c|c|}
\hline & \multirow[b]{2}{*}{$\begin{array}{l}\text { Male } \\
\text { population }\end{array}$} & \multicolumn{5}{|c|}{ Male_large clusters } & \multirow[b]{2}{*}{$\begin{array}{l}\text { Female } \\
\text { population }\end{array}$} & \multicolumn{5}{|c|}{ Female_large clusters } \\
\hline & & $\begin{array}{l}\text { MC1 } \\
\text { Beer/ } \\
\text { convenience } \\
(n=366)\end{array}$ & $\begin{array}{l}\text { MC2 } \\
\text { Traditional } \\
(n=192)\end{array}$ & $\begin{array}{l}\text { MC3 } \\
\text { Mixed/ } \\
\text { sweet } \\
(n=190)\end{array}$ & $\begin{array}{l}\text { MC4 } \\
\text { Healthier } \\
(n=185)\end{array}$ & Palue & & $\begin{array}{l}\text { FC1 } \\
\text { Traditional } \\
(n=355)\end{array}$ & $\begin{array}{l}F C 2 \\
\text { Healthier/ } \\
\text { cosmopolitan } \\
(n=275)\end{array}$ & $\begin{array}{l}\text { FC3 } \\
\text { Convenience } \\
(n=229)\end{array}$ & $\begin{array}{l}\text { FC4 } \\
\text { Healthier/ } \\
\text { sweet } \\
(n=168)\end{array}$ & Palue \\
\hline $\begin{array}{l}\text { Mean (SE) } \\
\text { kilojoules }\end{array}$ & $\begin{array}{l}10251 \\
(120)\end{array}$ & $\begin{array}{l}9199 \\
(130)\end{array}$ & $\begin{array}{l}11222 \\
(162)\end{array}$ & $\begin{array}{l}11011 \\
(157)\end{array}$ & $\begin{array}{l}9574 \\
(149)\end{array}$ & $\star \star \star$ & $\begin{array}{l}6929 \\
(100)\end{array}$ & $7316(98)$ & $\begin{array}{l}6889 \\
(106)\end{array}$ & $\begin{array}{l}6319 \\
(138)\end{array}$ & $\begin{array}{l}7193 \\
(103)\end{array}$ & $\star \star \star$ \\
\hline $\begin{array}{l}\text { Mean (SE) BMI } \\
\mathrm{kg} / \mathrm{m}^{2} \dagger\end{array}$ & $\begin{array}{l}24.9 \\
(0.11)\end{array}$ & $24.9(0.2)$ & $25.4(0.3)$ & $24.4(0.2)$ & $24.9(0.3)$ & $\star$ & $\begin{array}{l}24.6 \\
(0.15)\end{array}$ & $25.2(0.3)$ & $24.4(0.3)$ & $24.1(0.3)$ & $24.5(0.3)$ & $\star$ \\
\hline $\begin{array}{l}\text { \% "Low energy } \ddagger \\
\text { reporters" }\end{array}$ & 27 & 46.4 & 15.9 & 12.8 & 39.3 & $\star \star \star$ & 40 & 42.7 & 48.7 & 61.0 & 42.8 & $\star \star \star$ \\
\hline \% Slimmers & 4.2 & 3.6 & 3.1 & 4.2 & 7.0 & NS & 12.4 & 6.5 & 20.0 & 12.7 & 13.1 & 5 \\
\hline $\begin{array}{l}\% \text { Unwell and } \\
\text { eating affected }\end{array}$ & 4.7 & 8.2 & 1.6 & 4.2 & 2.2 & $\star \star \star$ & 9.5 & 8.7 & 10.5 & 14.4 & 4.8 & $\star \star \star$ \\
\hline
\end{tabular}

$\dagger \mathrm{BMI}=$ body mass index $\left(\mathrm{kg} / \mathrm{m}^{2}\right) . \ddagger “$ Low energy reporters” $=$ reported energy intake $\leqslant 1.2$ estimated basic metabolic rate $(\mathrm{BMR}) .{ }^{\star} \mathrm{p}<0.05 ;{ }^{\star \star} \mathrm{p}<0.01 ;{ }^{\star \star \star} \mathrm{p}<0.001$ ANOVA or $\chi^{2}$ test for the eight dietary clusters. Men and women separately.

reported median intakes in the "high" category for: white bread/refined cereals, butter and other margarines, tea and sugar/confectionery. Median intakes were moderately high for cakes/pastries, puddings, high fat dairy products and meat ham/bacon, potatoes and vegetables. Median intakes were "low" for wholegrain cereals and pasta/rice and zero for low fat dairy products, polyunsaturated spreads, wholegrain cereals, pasta/rice, fish/ shellfish, fruit juices, and liqueurs/wines/spirits. This dietary pattern could be termed a "traditional British diet".

Male cluster 3 (MC3) ( $=190 ; 17.5 \%$ of men) had median intakes in the "high" category for wholegrain cereals, fish/shellfish, fruits/nuts and cakes/pastries. There were moderately high intakes of high fat dairy products and coffee. In addition intake of polyunsaturated fats, low fat dairy products and liqueurs/spirits/wines were well above the respective population median values of zero. Cluster median intakes for fruit juices and other margarines were zero. This dietary pattern could be considered a "mixed sweet diet".
Male cluster 4 (MC4) $(n=185 ; 17 \%$ of men) had median intakes in the "high category" for wholegrain cereals, fish/shellfish, and fruit/ nuts. There were moderately high intakes of pasta/rice and coffee. In addition, median intakes of liqueurs/spirits/wines, low fat dairy products, polyunsaturated fat spreads and fruit juices were well above the respective population median of zero There was a zero median intake for "other margarines". This dietary pattern could be considered a "healthier diet".

WOMEN

The female cluster 1 (FC1) $(n=355 ; 32 \%$ of women) was the most prevalent dietary group (table 2). Reported food/drink groups with median intakes in the "high" category included: white bread/refined cereals, butter and high fat dairy products, and moderately high in tea, prepared meat products chips/roast potatoes, cakes/pastries, and preserves/confectionery. Alcoholic drinks and fruit juices were located in the "low" category, together with fish/shellfish, and low fat dairy products. The median intake of polyunsaturated fats/low fat spreads were zero. Intakes in the moderately low intake

Table 4 Macronutrient densities: four large male and female dietary clusters

\begin{tabular}{|c|c|c|c|c|c|c|c|c|c|c|c|c|}
\hline & \multirow[b]{2}{*}{$\begin{array}{l}\text { Male } \\
\text { population } \\
N=1087\end{array}$} & \multicolumn{5}{|c|}{ Male-large clusters } & \multirow[b]{2}{*}{$\begin{array}{l}\text { Female } \\
\text { population } \\
N=1100\end{array}$} & \multicolumn{5}{|c|}{ Female-large clusters } \\
\hline & & $\begin{array}{l}\text { MC1 } \\
\text { Beer/ } \\
\text { convenience } \\
(n=366)\end{array}$ & $\begin{array}{l}\text { MC2 } \\
\text { Traditional } \\
(n=192)\end{array}$ & $\begin{array}{l}\text { MC3 } \\
\text { Sweet/ } \\
\text { mixed } \\
(n=190)\end{array}$ & $\begin{array}{l}\text { MC4 } \\
\text { Healthy } \\
(n=185)\end{array}$ & $\stackrel{p}{\text { Value }}$ & & $\begin{array}{l}\text { FC1 } \\
\text { Traditional } \\
(n=355)\end{array}$ & $\begin{array}{l}F C 2 \\
\text { Healthy/ } \\
\text { cosmopolitan } \\
(n=275)\end{array}$ & $\begin{array}{l}\text { FC3 } \\
\text { Convenience } \\
(n=229)\end{array}$ & $\begin{array}{l}\text { FC4 } \\
\text { Healthy/ } \\
\text { e sweet } \\
(n=168)\end{array}$ & $\stackrel{p}{\text { Value }}$ \\
\hline $\begin{array}{l}\text { Alcohol \% TE† mean } \\
\text { (SE) }\end{array}$ & $\begin{array}{l}6.90 \\
(0.24)\end{array}$ & $\begin{array}{l}8.52 \\
(0.51)\end{array}$ & $\begin{array}{l}4.82 \\
(0.40)\end{array}$ & $\begin{array}{l}4.08 \\
(0.37)\end{array}$ & $\begin{array}{l}7.41 \\
(0.53)\end{array}$ & $\star \star \star \star ~$ & $2.8(0.12)$ & $\begin{array}{l}1.43 \\
(0.12)\end{array}$ & $\begin{array}{l}3.96 \\
(0.28)\end{array}$ & $2.0(0.19)$ & $\begin{array}{l}1.42 \\
(0.13)\end{array}$ & $\star \star \star \star$ \\
\hline $\begin{array}{l}\text { Total carbohydrate \% } \\
\text { FE } \ddagger \text { mean (SE) }\end{array}$ & $\begin{array}{l}46.09 \\
(0.29)\end{array}$ & $\begin{array}{l}45.09 \\
(0.50)\end{array}$ & $\begin{array}{l}48.09 \\
(0.47)\end{array}$ & $\begin{array}{l}47.96 \\
(0.60)\end{array}$ & $\begin{array}{l}44.83 \\
(0.79)\end{array}$ & $\star \star \star \star$ & $\begin{array}{l}46.62 \\
(0.22)\end{array}$ & $\begin{array}{l}47.08 \\
(0.39)\end{array}$ & $\begin{array}{l}45.60 \\
(0.46)\end{array}$ & $\begin{array}{l}47.69 \\
(0.44)\end{array}$ & $\begin{array}{l}46.81 \\
(0.59)\end{array}$ & 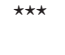 \\
\hline $\begin{array}{l}\text { Sugars }(\mathrm{g} / 1000 \text { kcals } \S) \\
\text { mean (SE) }\end{array}$ & $\begin{array}{l}49.72 \\
(0.47)\end{array}$ & $\begin{array}{l}45.10 \\
(0.85)\end{array}$ & $\begin{array}{l}49.99 \\
(1.09)\end{array}$ & $\begin{array}{l}54.11 \\
(0.95)\end{array}$ & $\begin{array}{l}50.10 \\
(1.09)\end{array}$ & $\star \star \star \star$ & $\begin{array}{l}51.88 \\
(0.47)\end{array}$ & $\begin{array}{l}50.17 \\
(0.89)\end{array}$ & $\begin{array}{l}52.73 \\
(0.86)\end{array}$ & $\begin{array}{l}52.34 \\
(1.17)\end{array}$ & $\begin{array}{l}53.79 \\
(1.04)\end{array}$ & $\star \star \star$ \\
\hline $\begin{array}{l}\text { Starch }(\mathrm{g} / 1000 \text { kcals } \S) \\
\quad \text { mean }(\mathrm{SE})\end{array}$ & $\begin{array}{l}68.44 \\
(0.38)\end{array}$ & $\begin{array}{l}70.12 \\
(0.71)\end{array}$ & $\begin{array}{l}70.02 \\
(0.76)\end{array}$ & $\begin{array}{l}68.22 \\
(0.77)\end{array}$ & $\begin{array}{l}66.88 \\
(0.90)\end{array}$ & $\star \star \star$ & $\begin{array}{l}65.12 \\
(0.37)\end{array}$ & $\begin{array}{l}67.65 \\
(0.62)\end{array}$ & $\begin{array}{l}61.96 \\
(0.74)\end{array}$ & $\begin{array}{l}66.13 \\
(0.91)\end{array}$ & $\begin{array}{l}64.33 \\
(0.77)\end{array}$ & $\star \star \star$ \\
\hline $\begin{array}{l}\text { Fibre }(\mathrm{g} / 1000 \mathrm{kcals} \rrbracket) \\
\text { mean }(\mathrm{SE})\end{array}$ & $\begin{array}{l}10.97 \\
(0.09)\end{array}$ & $\begin{array}{l}10.42 \\
(0.16)\end{array}$ & $\begin{array}{l}10.35 \\
(0.17)\end{array}$ & $\begin{array}{l}11.28 \\
(0.19)\end{array}$ & $\begin{array}{l}12.01 \\
(0.24)\end{array}$ & $\star \star \star$ & $\begin{array}{l}11.56 \\
(0.10)\end{array}$ & $\begin{array}{l}10.59 \\
(0.14)\end{array}$ & $\begin{array}{l}13.19 \\
(0.23)\end{array}$ & $\begin{array}{l}10.18 \\
(0.19)\end{array}$ & $\begin{array}{l}13.07 \\
(0.25)\end{array}$ & $\star \star \star$ \\
\hline Protein \% FE mean (SE) & $\begin{array}{l}15.19 \\
(0.09)\end{array}$ & $\begin{array}{l}15.70 \\
(0.20)\end{array}$ & $\begin{array}{l}14.35 \\
(0.18)\end{array}$ & $\begin{array}{l}14.29 \\
(0.14)\end{array}$ & $\begin{array}{l}16.39 \\
(0.23)\end{array}$ & $\star \star \star$ & $\begin{array}{l}15.61 \\
(0.10)\end{array}$ & $\begin{array}{l}14.66 \\
(0.15)\end{array}$ & $\begin{array}{l}17.32 \\
(0.24)\end{array}$ & $\begin{array}{l}14.86 \\
(0.27)\end{array}$ & $\begin{array}{l}15.86 \\
(0.21)\end{array}$ & $\star \star \star$ \\
\hline $\begin{array}{l}\text { Total fat \% FE mean } \\
\text { (SE) }\end{array}$ & $\begin{array}{l}40.39 \\
(0.14)\end{array}$ & $\begin{array}{l}41.01 \\
(0.28)\end{array}$ & $\begin{array}{l}40.42 \\
(0.31)\end{array}$ & $\begin{array}{l}39.74 \\
(0.29)\end{array}$ & $\begin{array}{l}39.65 \\
(0.32)\end{array}$ & $\star \star \star \star$ & $\begin{array}{l}40.32 \\
(0.14)\end{array}$ & $\begin{array}{l}40.93 \\
(0.27)\end{array}$ & $\begin{array}{l}39.44 \\
(0.34)\end{array}$ & $\begin{array}{l}40.57 \\
(0.36)\end{array}$ & $\begin{array}{l}39.67 \\
(0.39)\end{array}$ & $\star \star \star$ \\
\hline $\begin{array}{l}\text { Saturated and trans-fat \% } \\
\text { FE (mean SE) }\end{array}$ & $\begin{array}{l}18.72 \\
(0.09)\end{array}$ & $\begin{array}{l}18.65 \\
(0.18)\end{array}$ & $\begin{array}{l}19.83 \\
(0.21)\end{array}$ & $\begin{array}{l}18.79 \\
(0.20)\end{array}$ & $\begin{array}{l}17.78 \\
(0.20)\end{array}$ & $\star \star \star$ & $\begin{array}{l}18.72 \\
(0.09)\end{array}$ & $\begin{array}{l}20.28 \\
(0.19)\end{array}$ & $\begin{array}{l}18.15 \\
(0.21)\end{array}$ & $\begin{array}{l}18.71 \\
(0.23)\end{array}$ & $\begin{array}{l}18.58 \\
(0.22)\end{array}$ & $\star \star \star$ \\
\hline $\begin{array}{l}\text { Mono-fat \% FE mean } \\
\text { (SE) }\end{array}$ & $\begin{array}{l}12.43 \\
(0.06)\end{array}$ & $\begin{array}{l}13.05 \\
(0.11)\end{array}$ & $\begin{array}{l}12.41 \\
(0.13)\end{array}$ & $\begin{array}{l}11.75 \\
(0.12)\end{array}$ & $\begin{array}{l}11.99 \\
(0.13)\end{array}$ & $\star \star \star$ & $\begin{array}{l}12.43 \\
(0.06)\end{array}$ & $\begin{array}{l}12.47 \\
(0.10)\end{array}$ & $\begin{array}{l}11.94 \\
(0.13)\end{array}$ & $\begin{array}{l}12.59 \\
(0.14)\end{array}$ & $\begin{array}{l}11.29 \\
(0.14)\end{array}$ & $\star \star \star$ \\
\hline Poly-fat \% FE mean (SE) & $\begin{array}{l}6.22 \\
(0.07)\end{array}$ & $\begin{array}{l}6.24 \\
(0.11)\end{array}$ & $\begin{array}{l}5.21 \\
(0.12)\end{array}$ & $\begin{array}{l}6.20 \\
(0.17)\end{array}$ & $\begin{array}{l}6.89 \\
(0.18)\end{array}$ & $\star \star \star$ & $\begin{array}{l}6.02 \\
(0.06)\end{array}$ & $\begin{array}{l}5.19 \\
(0.08)\end{array}$ & $\begin{array}{l}6.36 \\
(13.31)\end{array}$ & $\begin{array}{l}6.37 \\
(0.14)\end{array}$ & $\begin{array}{l}6.83 \\
(0.21)\end{array}$ & $\star \star \star$ \\
\hline $\mathrm{P}: \mathrm{S}$ ratio $\|$ mean $(\mathrm{SE})$ & $\begin{array}{l}0.35 \\
(0.01)\end{array}$ & $\begin{array}{l}0.36 \\
(0.01)\end{array}$ & $\begin{array}{l}0.27 \\
(0.01)\end{array}$ & $\begin{array}{l}0.35 \\
(0.01)\end{array}$ & $\begin{array}{l}0.41 \\
(0.02)\end{array}$ & $\star \star \star$ & $\begin{array}{l}0.33 \\
(0.005)\end{array}$ & $\begin{array}{l}0.27 \\
(0.01)\end{array}$ & $\begin{array}{l}0.37 \\
(0.01)\end{array}$ & $\begin{array}{l}0.36 \\
(0.01)\end{array}$ & $\begin{array}{l}0.38 \\
(0.01)\end{array}$ & $\star \star \star$ \\
\hline
\end{tabular}

†TE $=$ total energy (food plus alcohol). $\ddagger \mathrm{FE}=$ food energy (excluding energy from alcohol). $\$ \mathrm{~g} / 1000 \mathrm{kcal}=\mathrm{g}$ per 1000 food kcal (excluding energy from alcohol). TPolyunsaturated to saturated and transfat ratio. ${ }^{\star \star \star} \mathrm{p}<0.001$ ANOVA for eight dietary clusters. Men and women separately. 
Table 5 Micronutrient densities: four large male and female dietary clusters

\begin{tabular}{|c|c|c|c|c|c|c|c|c|c|c|c|c|}
\hline & \multirow[b]{2}{*}{$\begin{array}{l}\text { Male } \\
\text { population }\end{array}$} & \multicolumn{5}{|c|}{ Male_large clusters } & \multirow[b]{2}{*}{$\begin{array}{l}\text { Female } \\
\text { population }\end{array}$} & \multicolumn{5}{|c|}{ Female—large clusters } \\
\hline & & $\begin{array}{l}\text { MC1 } \\
\text { Beer/ } \\
\text { convenience } \\
(n=366)\end{array}$ & $\begin{array}{l}\text { MC2 } \\
\text { Traditional } \\
(n=192)\end{array}$ & $\begin{array}{l}\text { MC3 } \\
\text { Sweet/ } \\
\text { mixed } \\
(n=190)\end{array}$ & $\begin{array}{l}\text { MC4 } \\
\text { Healthy } \\
(n=185)\end{array}$ & $\stackrel{p}{\text { Value }}$ & & $\begin{array}{l}\text { FC1 } \\
\text { Traditional } \\
(n=355)\end{array}$ & $\begin{array}{l}F C 2 \\
\text { Healthy/ } \\
\text { cosmopolitan } \\
(n=275)\end{array}$ & $\begin{array}{l}\text { FC3 } \\
\text { Convenience } \\
(n=229)\end{array}$ & $\begin{array}{l}\text { FC4 } \\
\text { Healthy/ } \\
\text { sweet } \\
(n=168)\end{array}$ & $\stackrel{p}{\text { Value }}$ \\
\hline $\begin{array}{l}\text { Retinol equivalents } \\
(\mu \mathrm{g})\end{array}$ & $683(25.5)$ & $\begin{array}{l}618.0 \\
(39.6)\end{array}$ & $\begin{array}{l}614.4 \\
(43.2)\end{array}$ & $\begin{array}{l}916.0 \\
(94.1)\end{array}$ & $\begin{array}{l}613.9 \\
(39.7)\end{array}$ & $\star \star \star \star$ & $869(33.6)$ & $631(680)$ & $\begin{array}{l}1191 \\
(1617)\end{array}$ & $678(924)$ & $\begin{array}{l}1056 \\
(1001)\end{array}$ & $\star \star \star \star$ \\
\hline Vitamin D $(\mu \mathrm{g})$ & $\begin{array}{l}1.42 \\
(0.04)\end{array}$ & $\begin{array}{l}1.17 \\
(0.09)\end{array}$ & $\begin{array}{l}1.39 \\
(0.07)\end{array}$ & $\begin{array}{l}1.72 \\
(0.07)\end{array}$ & $\begin{array}{l}1.74 \\
(0.11)\end{array}$ & $\star \star \star \star$ & $\begin{array}{l}1.54 \\
(0.04)\end{array}$ & $\begin{array}{l}1.38 \\
(0.05)\end{array}$ & $\begin{array}{l}1.62 \\
(0.08)\end{array}$ & $\begin{array}{l}1.33 \\
(0.11)\end{array}$ & $\begin{array}{l}2.17 \\
(0.12)\end{array}$ & $\star \star \star \star$ \\
\hline Vitamin E (mg) & $\begin{array}{l}4.08 \\
(0.05)\end{array}$ & $\begin{array}{l}3.89 \\
(0.10)\end{array}$ & $\begin{array}{l}3.37 \\
(0.08)\end{array}$ & $\begin{array}{l}4.42 \\
(0.12)\end{array}$ & $\begin{array}{l}4.56 \\
(0.12)\end{array}$ & $\star \star \star$ & $\begin{array}{l}4.29 \\
(0.05)\end{array}$ & $\begin{array}{l}3.73 \\
(0.06)\end{array}$ & $\begin{array}{l}4.69 \\
(0.10)\end{array}$ & $\begin{array}{l}4.23 \\
(0.13)\end{array}$ & $\begin{array}{l}5.14 \\
(0.14)\end{array}$ & $\star \star \star$ \\
\hline Vitamin C (mg) & $\begin{array}{l}27.9 \\
(0.52)\end{array}$ & $\begin{array}{l}23.93 \\
(0.79)\end{array}$ & $\begin{array}{l}21.51 \\
(0.66)\end{array}$ & $\begin{array}{l}27.12 \\
(0.85)\end{array}$ & $\begin{array}{l}39.87 \\
(1.67)\end{array}$ & $\star \star \star \star$ & $\begin{array}{l}38.2 \\
(0.78)\end{array}$ & $\begin{array}{l}28.58 \\
(0.83)\end{array}$ & $\begin{array}{l}52.82 \\
(1.92)\end{array}$ & $\begin{array}{l}33.54 \\
(1.78)\end{array}$ & $\begin{array}{l}42.50 \\
(1.77)\end{array}$ & $\star \star \star \star$ \\
\hline Thiamin (mg) & $\begin{array}{l}0.07 \\
(0.01)\end{array}$ & $\begin{array}{l}0.67 \\
(0.01)\end{array}$ & $\begin{array}{l}0.69 \\
(0.01)\end{array}$ & $\begin{array}{l}0.75 \\
(0.01)\end{array}$ & $\begin{array}{l}0.76 \\
(0.01)\end{array}$ & $\star \star \star \star$ & $\begin{array}{l}0.76 \\
(0.01)\end{array}$ & $\begin{array}{l}0.74 \\
(0.01)\end{array}$ & $\begin{array}{l}0.79 \\
(0.01)\end{array}$ & $\begin{array}{l}0.71 \\
(0.02)\end{array}$ & $\begin{array}{l}0.82 \\
(0.01)\end{array}$ & $\star \star \star \star$ \\
\hline Riboflavin (mg) & $\begin{array}{l}0.86 \\
(0.01)\end{array}$ & $\begin{array}{l}0.83 \\
(0.02)\end{array}$ & $\begin{array}{l}0.78 \\
(0.02)\end{array}$ & $\begin{array}{l}0.94 \\
(0.02)\end{array}$ & $\begin{array}{l}0.96 \\
(0.02)\end{array}$ & $\star \star \star$ & $\begin{array}{l}0.96 \\
(0.01)\end{array}$ & $\begin{array}{l}0.87 \\
(0.01)\end{array}$ & $\begin{array}{l}1.10 \\
(0.03)\end{array}$ & $\begin{array}{l}0.86 \\
(0.03)\end{array}$ & $\begin{array}{l}1.09 \\
(0.03)\end{array}$ & $\star \star \star$ \\
\hline $\begin{array}{l}\text { Niacin equivalents } \\
(\mathrm{mg})\end{array}$ & $\begin{array}{l}16.6 \\
(0.13)\end{array}$ & $\begin{array}{l}16.82 \\
(0.27)\end{array}$ & $\begin{array}{l}15.27 \\
(0.43)\end{array}$ & $\begin{array}{l}16.28 \\
(0.22)\end{array}$ & $\begin{array}{l}18.08 \\
(0.28)\end{array}$ & $\star \star \star \star$ & $\begin{array}{l}17.6 \\
(0.15)\end{array}$ & $\begin{array}{l}15.88 \\
(0.20)\end{array}$ & $\begin{array}{l}19.69 \\
(0.35)\end{array}$ & $\begin{array}{l}16.52 \\
(0.31)\end{array}$ & $\begin{array}{l}18.22 \\
(0.32)\end{array}$ & $\star \star \star \star$ \\
\hline Vitamin B6 (mg) & $\begin{array}{l}1.02 \\
(0.01)\end{array}$ & $\begin{array}{l}1.08 \\
(0.02)\end{array}$ & $\begin{array}{l}0.90 \\
(0.02)\end{array}$ & $\begin{array}{l}0.96 \\
(0.02)\end{array}$ & $\begin{array}{l}1.11 \\
(0.02)\end{array}$ & $\star \star \star \star$ & $\begin{array}{l}0.96 \\
(0.01)\end{array}$ & $\begin{array}{l}0.90 \\
(0.01)\end{array}$ & $\begin{array}{l}1.05 \\
(0.02)\end{array}$ & $\begin{array}{l}0.91 \\
(0.02)\end{array}$ & $\begin{array}{l}0.98 \\
(0.02)\end{array}$ & $\star \star \star \star$ \\
\hline Vitamin B12 $(\mu \mathrm{g})$ & $\begin{array}{l}3.02 \\
(0.07)\end{array}$ & $\begin{array}{l}2.99 \\
(0.11)\end{array}$ & $\begin{array}{l}2.64 \\
(0.12)\end{array}$ & $\begin{array}{l}3.42 \\
(0.26)\end{array}$ & $\begin{array}{l}3.06 \\
(0.14)\end{array}$ & $\star$ & $\begin{array}{l}3.23 \\
(0.09)\end{array}$ & $\begin{array}{l}2.60 \\
(0.10)\end{array}$ & $\begin{array}{l}4.12 \\
(0.22)\end{array}$ & $\begin{array}{l}2.87 \\
(0.19)\end{array}$ & $\begin{array}{l}3.51 \\
(0.21)\end{array}$ & $\star \star \star \star ~$ \\
\hline Folate $(\mu \mathrm{g})$ & $129(1.4)$ & $\begin{array}{l}133.34 \\
(3.53)\end{array}$ & $\begin{array}{l}116.47 \\
(1.98)\end{array}$ & $\begin{array}{l}120.89 \\
(1.97)\end{array}$ & $\begin{array}{l}141.39 \\
(2.58)\end{array}$ & $\star \star \star$ & $130(1.5)$ & $\begin{array}{l}113.4 \\
(1.73)\end{array}$ & $\begin{array}{l}151.2 \\
(3.10)\end{array}$ & $\begin{array}{l}120.8 \\
(4.20)\end{array}$ & $\begin{array}{l}143.3 \\
(3.26)\end{array}$ & $\star \star \star \star$ \\
\hline Biotin $(\mu \mathrm{g})$ & $\begin{array}{l}16.5 \\
(0.58)\end{array}$ & $\begin{array}{l}16.95 \\
(1.67)\end{array}$ & $\begin{array}{l}14.19 \\
(0.33)\end{array}$ & $\begin{array}{l}16.24 \\
(0.29)\end{array}$ & $\begin{array}{l}18.54 \\
(0.47)\end{array}$ & - & $\begin{array}{l}17.6 \\
(0.45)\end{array}$ & $\begin{array}{l}14.18 \\
(0.23)\end{array}$ & $\begin{array}{l}20.14 \\
(0.56)\end{array}$ & $\begin{array}{l}17.44 \\
(1.87)\end{array}$ & $\begin{array}{l}19.07 \\
(0.90)\end{array}$ & $\star \star \star \star$ \\
\hline $\begin{array}{l}\text { Pantothenic acid } \\
\text { (mg) }\end{array}$ & $\begin{array}{l}2.61 \\
(0.03)\end{array}$ & $\begin{array}{l}2.68 \\
(0.05)\end{array}$ & $\begin{array}{l}2.41 \\
(0.04)\end{array}$ & $\begin{array}{l}2.53 \\
(0.04)\end{array}$ & $\begin{array}{l}2.82 \\
(0.08)\end{array}$ & $\star \star \star \star$ & $\begin{array}{l}2.78 \\
(2.65)\end{array}$ & $\begin{array}{l}2.60 \\
(0.04)\end{array}$ & $\begin{array}{l}3.08 \\
(0.06)\end{array}$ & $\begin{array}{l}2.66 \\
(0.07)\end{array}$ & $\begin{array}{l}2.90 \\
(0.06)\end{array}$ & $\star \star \star \star$ \\
\hline Iron $(\mathrm{mg})$ & $\begin{array}{l}5.69 \\
(0.07)\end{array}$ & $\begin{array}{l}5.49 \\
(0.16)\end{array}$ & $\begin{array}{l}5.26 \\
(0.12)\end{array}$ & $\begin{array}{l}6.03 \\
(0.12)\end{array}$ & $\begin{array}{l}6.26 \\
(0.13)\end{array}$ & $\star \star \star \star$ & $6.4(0.08)$ & $\begin{array}{l}5.81 \\
(0.01)\end{array}$ & $\begin{array}{l}7.46 \\
(0.19)\end{array}$ & $\begin{array}{l}5.73 \\
(0.17)\end{array}$ & $\begin{array}{l}6.87 \\
(0.18)\end{array}$ & $\star \star \star \star$ \\
\hline Calcium (mg) & $386(3.5)$ & $\begin{array}{l}335.95 \\
(7.49)\end{array}$ & $\begin{array}{l}375.94 \\
(5.59)\end{array}$ & $\begin{array}{l}403.55 \\
(5.39)\end{array}$ & $\begin{array}{l}442.04 \\
(8.36)\end{array}$ & $\star \star \star$ & $437(4.4)$ & $\begin{array}{l}417.3 \\
(5.97)\end{array}$ & $\begin{array}{l}467.6 \\
(8.05)\end{array}$ & $\begin{array}{l}404.5 \\
(13.83)\end{array}$ & $\begin{array}{l}491.9 \\
(8.85)\end{array}$ & $\star \star \star \star$ \\
\hline Potassium (mg) & $1322(9.0)$ & $\begin{array}{l}1314.4 \\
(19.5)\end{array}$ & $\begin{array}{l}1238.3 \\
(15.8)\end{array}$ & $\begin{array}{l}1277.4 \\
(15.5)\end{array}$ & $\begin{array}{l}1457.4 \\
(17.3)\end{array}$ & $\star \star \star \star$ & $1485(12)$ & $\begin{array}{l}1345.8 \\
(15.5)\end{array}$ & $\begin{array}{l}1658.9 \\
(24.1)\end{array}$ & $\begin{array}{l}1385.4 \\
(29.8)\end{array}$ & $\begin{array}{l}1582.9 \\
(24.3)\end{array}$ & $\star \star \star \star$ \\
\hline Magnesium (mg) & $133(1.3)$ & $\begin{array}{l}130.5 \\
(3.07)\end{array}$ & $\begin{array}{l}118.8 \\
(1.87)\end{array}$ & $\begin{array}{l}131.5 \\
(1.85)\end{array}$ & $\begin{array}{l}148.5 \\
(1.97)\end{array}$ & $\star \star \star \star$ & $143(1.4)$ & $\begin{array}{l}124.2 \\
(1.71)\end{array}$ & $\begin{array}{l}164.8 \\
(2.56)\end{array}$ & $\begin{array}{l}133.4 \\
(3.68)\end{array}$ & $\begin{array}{l}157.6 \\
(3.04)\end{array}$ & $\star \star \star \star$ \\
\hline Phosphorus (mg) & $600(3.7)$ & $\begin{array}{l}585.9 \\
(7.69)\end{array}$ & $\begin{array}{l}557.8 \\
(5.96)\end{array}$ & $\begin{array}{l}603.7 \\
(6.20)\end{array}$ & $\begin{array}{l}667.9 \\
(8.46)\end{array}$ & $\star \star \star \star$ & $650(4.7)$ & $\begin{array}{l}598.5 \\
(5.95)\end{array}$ & $\begin{array}{l}725.8 \\
(9.19)\end{array}$ & $\begin{array}{l}600.00 \\
(12.56)\end{array}$ & $\begin{array}{l}717.4 \\
(10.5)\end{array}$ & $\star \star \star \star$ \\
\hline Copper (mg) & $\begin{array}{l}0.66 \\
(0.01)\end{array}$ & $\begin{array}{l}0.65 \\
(0.02)\end{array}$ & $\begin{array}{l}0.60 \\
(0.01)\end{array}$ & $\begin{array}{l}0.70 \\
(0.02)\end{array}$ & $\begin{array}{l}0.68 \\
(0.02)\end{array}$ & $\star \star \star \star$ & $\begin{array}{l}0.74 \\
(0.01)\end{array}$ & $\begin{array}{l}0.66 \\
(0.01)\end{array}$ & $\begin{array}{l}0.86 \\
(0.02)\end{array}$ & $\begin{array}{l}0.69 \\
(0.02)\end{array}$ & $\begin{array}{l}0.80 \\
(0.02)\end{array}$ & $\star \star \star \star$ \\
\hline Zinc (mg) & $\begin{array}{l}4.71 \\
(0.05)\end{array}$ & $\begin{array}{l}4.83 \\
(0.13)\end{array}$ & $\begin{array}{l}4.41 \\
(0.06)\end{array}$ & $\begin{array}{l}4.61 \\
(0.07)\end{array}$ & $\begin{array}{l}5.00 \\
(0.08)\end{array}$ & $\star \star \star$ & $\begin{array}{l}5.08 \\
(0.05)\end{array}$ & $\begin{array}{l}4.76 \\
(0.16)\end{array}$ & $\begin{array}{l}5.65 \\
(0.08)\end{array}$ & $\begin{array}{l}4.83 \\
(0.16)\end{array}$ & $\begin{array}{l}5.37 \\
(0.11)\end{array}$ & $\star \star \star \star$ \\
\hline Iodine $(\mu \mathrm{g})$ & $97(1.0)$ & $\begin{array}{l}98.38 \\
(1.93)\end{array}$ & $\begin{array}{l}89.96 \\
(2.93)\end{array}$ & $\begin{array}{l}94.11 \\
(1.70)\end{array}$ & $\begin{array}{l}105.4 \\
(1.92)\end{array}$ & $\star \star \star$ & $103(1.2)$ & $\begin{array}{l}98.86 \\
(2.17)\end{array}$ & $\begin{array}{l}106.98 \\
(2.09)\end{array}$ & $\begin{array}{l}97.61 \\
(3.02)\end{array}$ & $\begin{array}{l}112.96 \\
(2.65)\end{array}$ & $\star \star \star \star$ \\
\hline
\end{tabular}

category were: vegetables, fruit and wholegrain bread/cereals. This dietary pattern could be considered a "traditional British diet".

Female cluster 2 (FC2) ( $\mathrm{n}=275 ; 24.8 \%$ of women) was the second most prevalent dietary group (table 2). Reported median intakes were high for: wholegrain cereals, low fat dairy products, fish/shellfish, fruit/nuts and liqueurs/ spirits/wines. Median intakes of polyunsaturated fats and fruit juices were also above the respective population medians, of zero. Moderately high intakes included vegetables and salads, poultry, prepared meat products, pasta and coffee. "Low" median intakes were recorded for refined cereals and chips, while median intakes for beer/cider and other margarines were zero. This dietary pattern could be considered a "healthier cosmopolitan diet" (table 2).

Female cluster 3 (FC3) ( $=229 ; 21 \%$ of women) had reported median intakes in the "high" and "moderate-high" categories for: tea, pasta/rice, white bread/refined cereals, prepared meat products, chips/roast potatoes, sugar/confectionery, soft drinks while the median intake of "other margarines" was above the population median intake of zero. Food groups in the "low" category included low fat dairy products, wholegrain cereals, fish, fruits/ nuts, fruit juices, liqueurs/wines/spirits, beer/ cider. Median intakes of polyunsaturated fat/low fat spreads and fish/shellfish were zero. This dietary pattern could be considered a "convenience food diet" (table 2).

Female cluster 4 (FC4) ( $=168 ; 15 \%$ of women) had reported median intakes in the "high intake" category for: wholegrain cereals, low fat dairy products, fish/shellfish, fruits/ nuts, and median intakes of polyunsaturated fats and fruit juices were above the respective population medians of zero. Moderately high intakes included vegetables/salad, ham/bacon, cakes/pastries and puddings, biscuits and tea. Moderately low intakes included white bread/ refined cereals, and high fat dairy products, chips/roast potatoes, and soft drinks. Zero median intakes were reported for other margarines and beer/cider. This dietary pattern could be considered a "healthier but sweet diet" (table 2).

ENERGY INTAKE, BMI AND SLIMMING

Table 3 presents data for the four large male and female clusters on mean energy intake and body mass index (BMI), and the proportions of low energy reporters (LERs), self reported slimmers and individuals reporting that their diet was affected because of ill health during the survey week. Among both men and women, cluster differences in energy intakes, BMI, \% LERs and \% reporting that their diet had been affected by ill health were statistically significant at $\mathrm{p}<0.05$ to $\mathrm{p}<0.001$ ). 
Among the four large clusters, the "convenience food diet" (MC1; FC3) and "healthier diets" (MC4; FC2) had the lowest mean reported energy intakes, and for men the "convenience diet" had the highest proportion of LERs. Among women, the proportion of LERs was over $40 \%$ in all four large clusters, but was highest $(61 \%)$ in the "convenience food diet" (FC3). Among both men and women the "traditional diet" (MC2; FC1) had the highest mean energy intake, while the "healthier" (MC4; FC2) had the highest proportions of self reported slimmers, and the "convenience diet" (MC1; FC3) had the highest proportion reporting that their diet was affected because of ill health.

\section{MACRONUTRIENT DENSITY}

Table 4 presents data on macronutrient densities for the four large male and female clusters. Of the four large clusters, the "healthier diets" (MC4; FC2) had the lowest mean total fat, and saturated and trans fat, carbohydrate and starch. MC4 also had the highest polyunsaturated fat density and P:S ratio among men. Conversely, the "convenience diets" (MC1; FC3) and the "traditional diet" (MC2; FC1) had the highest mean densities of total fat and starch and the lowest densities of fibre among the four large clusters. Among the large clusters, the highest mean sugar densities were in the "mixed but sweet" and the "healthy sweet" dietary groups (MC3;FC4), which also had the highest polyunsaturated fat density (table 4).

\section{MICRONUTRIENT DENSITY}

Table 5 presents data on 20 micronutrients for the four large clusters for men and women. Of the four large clusters, the male and female "healthier diets" (MC4; FC2) had the highest mean micronutrient densities for 17 of 20 and 15 of 20 micronutrients respectively. The "traditional diet" clusters had the lowest mean densities for 16 of 20 micronutrients among
KEY POINTS

- Cluster analysis can identify groups within the UK population who report similar patterns of diet.

- $93 \%$ of men and $86 \%$ of women fall into one of four distinct diet groups; "beer and convenience food",

"traditional", "healthier but sweet" or "healthier" in men and "traditional", "healthier cosmopolitan", "convenience food" and "healthier but sweet" in women.

- Members of the diet groups exhibit different sociodemographic and behavioural characteristics and there are important differences in nutrient profiles between groups.

- Diet groups provide a more logical basis for developing and targeting public health nutrition policy than focusing on intakes of particular nutrients or foodstuffs.

men (MC2) and 14 of 20 micronutrients among women (FC1).

SOCIODEMOGRAPHIC AND BEHAVIOURAL

CHARACTERISTICS

Selected sociodemographic and behavioural characteristics for the four large dietary clusters for men and women are presented in table 6 . For both men and women, cluster differences for all variables examined were significant.

Compared with population averages, the "convenience" (MC1; FC3), and "traditional" diets (MC2; FC1) were characterised by higher proportions from the manual social classes and/or in receipt of State benefits. Higher proportions from both clusters smoked and among women fewer took micronutrient supplements. The "convenience diet" clusters (MC1; FC3) were on average younger, and had higher proportions of single people from ethnic minorities. Higher proportions from $\mathrm{MC} 1$

Table 6 Sociodemographic and behavioural profile: four large male and female clusters

\begin{tabular}{|c|c|c|c|c|c|c|c|c|c|c|c|c|}
\hline & \multirow[b]{2}{*}{$\begin{array}{l}\text { Male } \\
\text { population }\end{array}$} & \multicolumn{5}{|c|}{ Male_large clusters } & \multirow[b]{2}{*}{$\begin{array}{l}\text { Female } \\
\text { population }\end{array}$} & \multicolumn{5}{|c|}{ Female-large clusters } \\
\hline & & $\begin{array}{l}\text { MC1 Beer/ } \\
\text { convenience } \\
(n=366)\end{array}$ & $\begin{array}{l}\text { MC2 } \\
\text { Traditional } \\
(n=192)\end{array}$ & $\begin{array}{l}\text { MC3 } \\
\text { Sweet/mixed } \\
(n=190)\end{array}$ & $\begin{array}{l}\text { MC4 } \\
\text { Healthy } \\
(n=185)\end{array}$ & $p$ Value & & $\begin{array}{l}\text { FC1 } \\
\text { Traditional } \\
(n=355)\end{array}$ & $\begin{array}{l}\text { FC2 } \\
\text { Healthy/ } \\
\text { cosmopolitan } \\
(n=275)\end{array}$ & $\begin{array}{l}\text { FC3 } \\
\text { Convenience } \\
(n=229)\end{array}$ & $\begin{array}{l}\text { FC4 } \\
\text { Healthy/sweet } \\
(n=168)\end{array}$ & $\stackrel{p}{\text { Value }}$ \\
\hline \multicolumn{13}{|c|}{ Social class of informant (\%) } \\
\hline I, II & 35 & 26 & 22 & 41 & 53 & $\star \star \star \star$ & 35 & 12 & 35 & 15 & 31 & \\
\hline III NM & 13 & 14 & 10 & 14 & 12 & & 13 & 37 & 43 & 43 & 42 & $\star \star \star$ \\
\hline III M & 35 & 42 & 48 & 28 & 26 & & 31 & 9 & 7 & 7 & 7 & \\
\hline IV, V & 16 & 23 & 19 & 18 & 9 & & 20 & 42 & 15 & 36 & 20 & \\
\hline$\%$ Receiving benefits & 13 & 17 & 11 & 10 & 6 & $\star \star \star$ & 12 & 18 & 7 & 20 & 10 & $\star \star \star$ \\
\hline$\%$ White & 96 & 93 & 99 & 96 & 96 & $\star$ & 96 & 98 & 94 & 91 & 99 & $\star$ \\
\hline \multicolumn{13}{|l|}{ Region (\%) } \\
\hline Scotland & 9 & 10 & 6 & 5 & 10 & $\star \star$ & 9 & 11 & 7 & 10 & 6 & * \\
\hline Northern & 25 & 31 & 22 & 23 & 24 & & 26 & 26 & 24 & 25 & 29 & \\
\hline Central, SW Wales & 34 & 30 & 40 & 35 & 38 & & 33 & 36 & 31 & 28 & 35 & \\
\hline London SE & 33 & 30 & 31 & 39 & 29 & & 32 & 27 & 39 & 36 & 30 & \\
\hline$\%$ Cigarette smokers & 33.2 & 43 & 43 & 21 & 23 & $\star \star \star$ & 34.0 & 42 & 25 & 42 & 19 & $\star \star \star \star ~$ \\
\hline $\begin{array}{l}\% \text { Food supplement } \\
\text { users }\end{array}$ & 8.6 & 7 & 7 & 7 & 11 & $\star \star \star$ & 17.0 & 13 & 21 & 15 & 26 & $\star \star$ \\
\hline Age $(y)$ mean $(\mathrm{SE})$ & $50(10.2)$ & $\begin{array}{l}34.6 \\
(13.1)\end{array}$ & $\begin{array}{l}44.3 \\
(12.8)\end{array}$ & $\begin{array}{l}41.3 \\
(14.1)\end{array}$ & $\begin{array}{l}38.7 \\
(13.3)\end{array}$ & $\star \star \star$ & $\begin{array}{l}48 \\
(10.4)\end{array}$ & $\begin{array}{l}39.1 \\
(10.2)\end{array}$ & $\begin{array}{l}40.4 \\
(13.1)\end{array}$ & $\begin{array}{l}32.9 \\
(12.6)\end{array}$ & $\begin{array}{l}45.7 \\
(15.6)\end{array}$ & $\star \star \star$ \\
\hline \multicolumn{13}{|l|}{ Marital status (\%) } \\
\hline Married & 67 & 56 & 78 & 74 & 73 & & 68.9 & 69 & 74 & 54 & 81 & $\star \star \star$ \\
\hline Single & 27 & 35 & 17 & 23 & 23 & $\star \star \star$ & 17.0 & 17 & 14 & 28 & 8 & \\
\hline Separated/widowed & 6 & 9 & 5 & 4 & 4 & & 14.1 & 15 & 12 & 18 & 11 & \\
\hline
\end{tabular}


came from the North and Scotland whereas higher proportions from FC3 came from London and the South East. In contrast, higher proportions of the "healthier diet" clusters (MC4; FC2) and "mixed but sweet diet" and the "healthy sweet diet" clusters (MC3; FC4) were from the non-manual social classes, and were non-smokers compared with the respective population means. Compared with the population average, higher proportions of the male "healthier eaters" (MC4) were from Central/South West and Wales, and the "healthier but sweet" MC3 from London and the South East. Among women, higher proportions of "healthy eaters" (FC2) were from London and the South East.

\section{Discussion}

This paper uses cluster analysis to identify groups within the British population who report similar patterns of diet. Such an approach is potentially of relevance to public health nutrition policy as people eat foods not individual nutrients. There are a number of reasons why epidemiologists may wish to characterise specific food patterns, rather than focus on nutrients. Firstly, foods are not fully represented by their nutrient composition. Secondly, it is not possible to characterise the health effects associated with a given food solely on the basis of its nutrient content; this is because different components of a particular meal may compete with, antagonise, or change the bioavailabilty of any single nutrient contained in that meal. Thirdly, it can be difficult to disentangle the effects of various nutrients on health outcomes because of problems of multicollinearity. Finally, analyses based on foods are more directly related to dietary recommendations, and it becomes more feasible to identify practices that need to be modified in order to improve health outcomes.

Cluster analysis has not been widely used in public health nutrition. Probably the earliest example is the work of Akin $e t a l^{17}$ using data on adults aged $65-74$ years from the US Nationwide Food Consumption Survey and identifying dietary clusters with important differences in terms of nutrient intakes and socioeconomic characteristics. Subsequently, Tucker et $a l^{18}$ used cluster analysis on data derived from three day dietary records 680 elderly residents from the Boston area. In this study there were four major diet clusters, again with distinctive nutrient and socioeconomic characteristics. Of particular interest in this study was the finding that about half of the sample occupied a diet cluster high in intakes of milk, cereals and fruit, and with a favourable micronutrient intake and haematological profile.

In our study eight patterns of diet were identified in British men and women aged 16-64. Among men the most prevalent diet was a "beer and convenience diet" (34\% of the male population); second was a "traditional British diet" $(18 \%)$; third was "mixed but sweet diet" $(17.5 \%)$; and fourth was a "healthier diet" $(17 \%)$. Among women, the most prevalent diet was a " traditional British diet" (32\%); then a "healthy cosmopolitan diet" (25\%); thirdly a "convenience food diet" (21\%); and finally a "healthier but sweet diet" (15\%).

Hulshof et $a l^{19}$ carried out a comparable study, analysing data from the Dutch Nutritional Surveillance System, which is based on two day food records collected from 3781 adults (1802 men and 1979 women) during 1987-88. Eight dietary clusters were found, four of which were regarded as of poor nutritional quality on the basis of a scoring system for percentage saturated fat, P:S ratio, cholesterol, dietary fibre, alcohol and percentage mono/disaccharides. Another interesting paper is that of Haines et $a l,{ }^{17}$ which identifies nutrient differences among American women clustered according to possible food consumption locations. In this study, the "fast food" group are characterised by high intakes of fat, cholesterol and sodium, compared with a "home mixed eating" group with the most healthy diets and the authors emphasise the importance of targeting food messages to eating environments as well as food choices. ${ }^{20}$

Diet groups derived from cluster analysis might well predict important disease outcomes, such as cancer, heart disease of hypertension. Huijbregts et $a l^{4}$ used cluster analysis of food scores to assess relations with cardiovascular risk factors. The high alcohol intake group was associated with cardiovascular risk factors, but the healthy diet group was associated with more favourable levels of cardiovascular risk factors. Nicklas et al used factor analysis to assess eating patterns and their relation to cardiovascular risk factors. Eating patterns were associated with HDL-C, VLDL-C, triglycerides and systolic blood pressure. Farchi et $a l^{\beta}$ used a cluster technique to group Italian adults on the basis of their macronutrient intake. Four groups were identified that differed in terms of mortality from coronary heart disease, cancer and cirrhosis of the liver.

Lifestyle variables such as smoking and poor diet can independently affect health status and a combination of lifestyle practices may introduce a health risk that is greater than would be expected from the sum of individual risk factors. ${ }^{21}$ In our study there were important differences in sociodemographic and behavioural characteristics across the diet clusters. For example, a high prevalence of smoking and manual social class were characteristic of the "beer and convenience/convenience diet" and "traditional diet" groups in men and women. Similar observations were made in the study of Hulshof et al. ${ }^{19}$ On the basis of extensive epidemiological data published elsewhere, this mix of risk factors would be expected to confer high cardiovascular disease and cancer risks in associated diet clusters. ${ }^{21} 22$

We believe our results could be of relevance to the development, monitoring and targeting of public health nutrition policy in the UK. British populations do not meet dietary targets. ${ }^{23}$ Nevertheless, a public health strategy based on mass population change may be ill conceived. We have demonstrated that cluster analysis based on dietary intake is a useful tool 
for identifying risk groups within the population. Clusters differ in multi-dimensional ways and the determinants of food habits and lifestyles are also likely to vary. Factors such as convenience, cost, peer group pressure and occupation may be relevant to food choices and lifestyle. We would recommend targeting risk groups with appropriate health promotion strategies. Further research is needed on levers and barriers to change in these distinctive groups and programmes need to be appropriately tailored for maximum impact.

Funding: Department of Health

Conflicts of interest: none.

\section{Appendix}

A list of the $51 \mathrm{food} /$ beverage groups (Gregory et $a l^{10}$ pages $376-8)$

1 Pasta, rice and other miscellaneous cereals

2 White bread

3 Wholemeal bread

4 Other breads (rye, crumpets, muffins, pikelets, granary rolls and brown bread.)

5 Wholegrain and high fibre breakfast cereals ( All Bran, Branflakes, Shredded Wheat, museli, Weetabix)

6 Other breakfast cereals (Cornflakes, Rice Krispies, Special K, Sugar Puffs, Honey Smacks).

7 Biscuits (sweet and savoury)

8 Buns, cakes, pastries and fruit pies (Danish pastry, Chelsea bun, doughnuts, Eccles cakes, frangipane tarts, jam tarts, scones, sponge cakes, fruit cakes, meringue, fruit pies).

9 Puddings including ice cream (instant whip, fruit crumble, artic roll, batter puddings, custard/ blancmange, christmas pudding, fruit flans, fresh cream desserts, jelly, fruit fools, sponge puddings, milk puddings, sorbets, ice cream).

10 Whole milk (includes pasteurised, UHT, sterilised, Channel Island).

11 Semi-skimmed milk (pasteurised, UHT, flavoured, canned, milk with added vitamins)

12 Skimmed milk (pasteurised, UHT, sterilised, canned, milk with added vitamins).

13 Other milk and cream (condensed, dried milks, evaporated, goats milk, sheep milk, soya, milk shakes and all cream)

14 Cheese (hard, soft, cream cheese, processed cheese, cottage, and curd cheese).

15 Yoghurt (low fat, thick and creamy, "diet" yoghurts, goats and sheep milk yoghurt and yoghurt drinks).

16 Eggs and egg dishes (boiled, scrambled, omlettes, souffle, quiche, flans, Scotch eggs).

17 Butter

18 Polyunsaturated margarine (high in polyunsaturated fatty acids)

19 Low fat spreads.

20 Block margarine

21 Other margarine and spreads (soft margarine not polyunsaturated and yellow spreads).

22 Bacon and ham (bacon joints and rashers, gammon joints/steaks, ham sliced off the bone).

23 Beef, veal and dishes (beef and veal, joints, steaks, minced beef, stewing steak, beef stews and casseroles, meat balls, lasagne, chilli con carne, beef curry dishes, bolognaise sauce)

24 Lamb and dishes (lamb joints, chops, lamb curries, Irish stews, lamb casseroles/ stews).

25 Pork and dishes (joints, chops, steaks, belly rashers, pork stews/ casseroles, sweet and sour pork, spare ribs).

26 Coated chicken (chicken and turkey drumsticks, chicken pieces, nuggetts, fingers, burgers, etc, coated in egg and crumb, Kentucky fried chicken).

27 Chicken and turkey dishes ( roast chicken and turkey, barbecued, fried (no coating) pieces, curries, stews/ casseroles, chow mein, in sauce, spread, chicken/turkey roll)
28 Liver, liver pate, etc (all types of liver fried, stewed and grilled, liver casserole, liver sausage).

29 Burgers and kebabs (beef burgers, ham burgers, cheese burgers, doner/shish/kofte kebabs).

30 Sausages (beef, pork, turkey, polony, sausage in batter, saveloy, frankfurters).

31 Meat pies and pastries (chicken/turkey pies, vol-auvents, beef pies, pork pies, veal and ham pies, pasties, sausage rolls, meat samosas).

32 Other meat and meat products (game, rabbit, offal, faggots, black pudding, meat paste, canned meats, salami, meat loaf, moussaka, shepherds pie, chop suey).

33 White fish in batter of breadcrumbs (cod, haddock, hake, plaice, dogfish, skate,scampi, fish cakes, fish fingers cod roe fried, prawn balls).

34 Other white fish (cod, haddock, hake, plaice, shellfish, curried fish, fish paste, fish in sauce, fish pie, kedgeree).

35 Oily fish (herrings, kippers,mackererel, sprats, eels, herring roe, salmon, tuna, sardines, taramasalata, mackerel paste).

36 Salad vegetables (endive, lettuce, spinach, chicory, other raw vegetables cabbage,carrots, tomatoes, radish, spring onions, coleslaw and prepared salads).

37 Vegetables (beans/ pulses, cooked vegetables, vegetable casseroles/ stews, vegetable curries, torfu, ratatouille, cauliflower cheese).

38 Fried potatoes and chips (fresh or frozen chips, oven chips, potato waffles, hash browns, roast, sauteed, croquettes)

39 Other potatoes (boiled, mashed, jacket, potato salad, canned potato, potato based curries, instant potato).

40 Fruit and nuts (fruit cooked, raw, canned, fruit pie fillings, nuts including almonds, hazelnuts, mixed nuts, peanuts, peanut butter, bombay mix, seeds).

41 Sugar and preserves (white and brown sugar, glucose, black molasses, treacle, syrup, jams, marmalade, glace cherries, mixed peel, marzipan)

42 Savoury snacks (crisps, puffs, rings, twiglets).

43 Confectionery (boiled sweets, gums, pastils, fudge, chews, mints, rock, liquorice, toffees, popcorn)

44 Confectionery chocolate (chocolate bars, filled bars, assortments)

45 Fruit juice (single fruit juice and mixed fruit juice ; canned, bottled, cartons, carbonated, still, freshly squeezed)

46 Soft drinks (carbonated and low calorie versions, fruit squashes, cordials, fruit drinks, syrups)

47 Spirits and liquors (cream liquors, pernod, Southern Comfort, $70 \%$ proof spirits, Pimms)

48 Wine (white, red rose, sparkling, champagne, port, sherry)

49 Beer/cider (beer, lager, low alcohol stout, strong ale, cider, babycham, perry)

50 Miscelleneous (sauces, ketchups, chutneys, pickles, gravy, mayonnaise, soups, beverages (not tea of coffee).

51 Tea, coffee and water (instant and leaf/bean, also lemon tea, vending machine tea and coffee; tap water, bottled, carbonated

1 Schwerin HS, Stanton JL, Smith JL, et al. Food, eating habits and health: a further examination of relationships between food eating patterns and nutritional health. Am f Clin Nutr 1982;35:1319-25.

2 Randall E, Marshall JR, Grahman S, et al. Patterns of food use and their associations with nutrient Intakes. Am $\mathcal{F}$ Clin

3 Farchi G, Mariotti S, Menotti A, et al. Diet and 20 year mortality in two rural population groups of middle-aged men in Italy. Am F Clin Nutr1989;50:1095-110.

4 Huijbregts PCW, Feskens EJM, Kromhout D. Dietary patterns and cardiovascular risk factors in elderly men: The Zutphen Elderly Study. Int F Epidemiol 1995;24:313-20.

5 Campbell C, Roe DA, Eickwork K. Qualitative diet indexes: a descriptive or an assessment tool? 7 Am Diet Assoc 1982; 81:687-94.

6 Krebs-Smith SM, Smiciklas-Wright H, Gunthie HA.. The effect of variety in food choices on dietary quality. $f \mathrm{Am}$ Diet Assoc 1987;87:897-903.

7 Nicklas TA, Webber LS, Thompson B, et al. A mutlivariate model for assessing eating patterns and their relationships to cardiovascular risk factors: The Bogalusa Heart Study. Am f Clin Nutr 1989;49:1320-7. 
8 Barker ME, McClean KA, Thompson KA, et al. Dietary behaviours and sociocultural demographics in Northern Ireland. Br f Nutr 1990;64:319-29.

9 Gex-Fabry M, Raymond L, Jeanneret O. Multivariate analysis of dietary patterns of 939 Swiss adults: sociodemographic parameters and alcohol consumption profiles. Int $\mathcal{F}$ Epidemiol 1988;17:548-55.

10 Gregory J, Foster $\mathrm{K}$, Tyler $\mathrm{H}$, et al. The Dietary and Nutritional Survey of Adults. London: HMSO, 1990.

11 Kish L. Survey sampling. London: Wiley, 1965.

12 Everitt B. Cluster analysis. London: Heineman Educational Books, 1980

13 Blashfield RK. Mixture model tests of cluster analysis: accuracy of four agglomerative hierarchical methods. Psychol Bull1976;83:377-88.

14 Edelbrock C. Mixture model tests of hierarchical clustering algorithms: The problem of classifying everybody. Multi-
variate Behaviour Research 1979;14:367-84.

15 Pryer JA, Vrijheid M, Nichols R, et al. Who are the "low energy reporters" in the Dietary and Nutritional Survey of energy reporters" in the Dietary and Nutritiona

16 World Health Organisation. Energy and Protein Requirements. Rechnical Report Series no 724 . Geneva: WHO, 1985 .
17 Akin JS, Guilty DK, Popkin BM, et al. Cluster analysis of food consumption patterns of older Americans. $\mathcal{F}$ Am Diet food consumption patter

18 Tucker KL, Dallal GE, Rush D. Dietary patterns of the eldrly Boston-area residents defined by cluster analysis. $\mathcal{F} \mathrm{Am}$ Diet Assoc 1992;92:1487-91.

19 Hulshof KFAM, Wedel M, Lewik MRH, et al. Clustering of dietary variables and other lifestyle factors (Dutch Nutritional Surveillance System). I Epidemiol Community Health 1992;46:417-24.

20 Haines P, Hungerford BW, Popkin BW, et al. Eating patterns and energy and nutrient intakes of US women. 7 Am Diet Assoc 1992;92:698-704.

21 Marmot M, Elliott P, eds. Coronary heart disease epidemiology. From aetiology to public health. Oxford: Oxford University Press, 1992.

22 World Cancer Research Fund. Food, nutrition and the prevention of cancer : a global perspective. Washington DC: Amerition of cancer: a global perspective. Wash

23 Pryer JA, Brunner E, Elliott P, et al. Who complies with dietary recommendations for the prevention of coronary heart disease, and what do they eat? Findings from the Dietary and Nutritional Survey of British Adults. Eur $\mathcal{F}$ Clin Nutr 1995;49:718-28. 